\title{
The Effect of Staging Laparoscopy Prior to Neoadjuvant Chemotherapy on Treatment Management in Locally Advanced Gastric Cancer
}

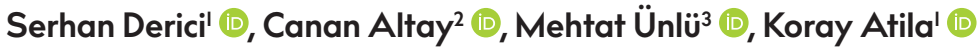 \\ 'Department of General Surgery, Dokuz Eylül University School of Medicine, İzmir, Turkey \\ 2Department of Radiology, Dokuz Eylül University School of Medicine, İzmir, Turkey \\ ${ }^{3}$ Department of Pathology, Dokuz Eylül University School of Medicine, İzmir, Turkey
}

ORCID IDs of the authors: S.D. 0000-0002-2828-I452; C.A. 0000-0003-04I7-7770; M.Ü. 0000-0002-7170-7594; K.A. 0000-000I-96285300 .

Cite this article as: Derici S, Altay C, Ünlü M, Atila K. The Effect of Staging Laparoscopy Prior to Neoadjuvant Chemotherapy on Treatment Management in Locally Advanced Gastric Cancer. Cyprus J Med Sci 2019; 4(2): 95-8.

\section{BACKGROUND/AIMS}

The prevalence of gastric adenocarcinoma is 951.000 in the world and cases in Western countries are diagnosed in a more advanced stage. In this study, the efficacy of neoadjuvant chemotherapy in gastric cancer was demonstrated and neoadjuvant chemotherapy was recommended for patients with $\geq T 2$ resectable gastric tumors. The key point for administering neoadjuvant chemotherapy is the distant location of a solid organ and the status of peritoneal metastasis at the beginning of the treatment. We aimed to investigate the effect of the peritoneal status, which is assessed using staging laparoscopy, on the treatment protocol of gastric cancer patients.

\section{MATERIAL and METHODS}

This retrospective study included 60 neoadjuvant chemotherapy patients, who were divided into 2 groups according to staging methods.

\section{RESULTS}

Out of a total of 60 patients, 30 were staged by radiological methods and staging laparoscopy prior to neoadjuvant chemotherapy. The remaining 30 patients were staged only by radiological methods. In the laparoscopic staged group, peritoneal metastases were detected in $43 \%$ of radiologically non-metastatic patients by staging laparoscopy. In the non-laparoscopic staged group, metastatic disease was identified in 7 patients, right before or during the gastrectomy operation.

\section{CONCLUSION}

Peritoneal metastasis is not uncommon in gastric cancer. If the evaluation of peritoneal status is made only by radiological examinations, the treatment may be started with an incorrect low-grade diagnosis. The use of staging laparoscopy prior to neoadjuvant chemotherapy makes a significant contribution to the multidisciplinary treatment of gastric cancer.

Keywords: Cancer staging, gastric cancer, Laparoscopy, neoadjuvant therapy, peritoneal carcinomatosis

\section{INTRODUCTION}

The prevalence of gastric adenocarcinoma is 951.000 in the world and the prevalence in underdeveloped countries is estimated to be 677.000 (I). The cases in Western countries are diagnosed in a more advanced stage of the disease, unlike the countries in the Far East (2). The incidence of gastric cancer in our country's region is found to be more similar to Far Eastern countries than to European and North American countries (I). Effective treatment methods should be discussed on priority for patients with advanced stage gastric cancer, especially in our region.

The efficacy of neoadjuvant chemotherapy in advanced gastric cancer patients was demonstrated in Western origin studies (3). Neoadjuvant chemotherapy is recommended for patients who have T2 and above resectable gastric tumors and no distant metastasis or peritoneal spread findings (4).

We aimed to investigate the effect of the peritoneal status, which is assessed using staging laparoscopy, on the treatment protocol of gastric cancer patients. 


\section{MATERIAL and METHODS}

\section{Patients}

Between August 2015 and September 2018, 1283 presentations registered to Dokuz Eylül University, Faculty of Medicine Upper Gastrointestinal (GI) were examined retrospectively at a case discussion meeting. Gastric cancer patients who had undergone a neoadjuvant chemotherapy plan were identified. Twenty-six patients were excluded from the study due to the lack of data.

\section{Staging, Chemotherapy, and Surgery}

The radiologic staging routinely was performed with computed tomography (CT) scanning. In both groups, patients with T2 or higher tumors and/or metastatic lymph nodes were considered to meet the criteria for neoadjuvant chemotherapy. All patients underwent the same neoadjuvant chemotherapy regimen. The second radiologic staging was performed in the third or fourth week after the previous neoadjuvant chemotherapy session. Surgery was performed at least 4 weeks later, following the last chemotherapy session.

The 60 consecutive patients with complete data were divided into 2 equal groups according to the staging methods. In the first group, patients were evaluated without using staging laparoscopy. The second group consisted of patients who underwent staging laparoscopy procedure prior to neoadjuvant chemotherapy.

\section{Staging Laparoscopy Technique}

Under general anesthesia, an infra-umbilical camera port and a right upper quadrant five-millimeter working port were placed in the supine position. The anterior abdominal wall, bilateral diaphragmatic surfaces, pelvic peritoneal, rectovesical/rectouterine region, omentum, and gastric wall were inspected. The location of the tumor, its mobilization, and its relation to the serosa and other organs were examined. The liver, spleen, jejunum, ileum, cecum, colon, and their mesenteric structures were examined. If an implant was detected, it was excised and then fixed with formaldehyde solution. If acid was found, it was aspirated and examined by cytological methods. In each case, the abdom- inal cavity was washed with $1000 \mathrm{ml}$ physiologic normal saline. By changing the position of the operating table, it was ensured that the fluid would come into contact with the entire abdominal wall, small intestines, omentum, diaphragmatic surfaces, pelvis, and abdominal organs. Then, the washing liquid was aspirated. This aspirated liquid was centrifuged and the precipitate was obtained. Two smears were prepared for pathological examination; one of them was stained with hematoxylin eosin and the other was stained with Papanicolaou stain.

This research was conducted according to the principles of the World Medical Association's Declaration of Helsinki, "Ethical Principles for Medical Research Involving Human Subjects" (amended in October 2013). Ethics committee approval was received for this study from Dokuz Eylül University (Approval Date: 23.01.2019, Approval Number: 2019/02-05). Oral informed consent was obtained from all the patients.

\section{Statistical Analysis}

Statistical analysis was performed using the Statistical Package for the Social Sciences program Statistical Package for the Social Sciences version 22.0 (IBM Corp., Armonk, NY, USA). Data were expressed as mean $\pm S D$ or median (minimum-maximum). Frequencies and percentages were used to depict categorical variables. A p-value of $<0.05$ was considered statistically significant.

\section{RESULTS}

The median age of the patients was 62 (37-84) years. Fifteen patients (25\%) were female and 45 (75\%) were male. Thirty patients underwent neoadjuvant chemotherapy without staging laparoscopy (non-laparoscopic staged group, GROUP I), and 30 patients underwent staging laparoscopy prior to neoadjuvant chemotherapy (laparoscopic staged group, GROUP 2). No gender-based significance was detected between the groups (Group I: 25 males $\alpha 5$ females; Group 2: 20 males $\alpha$ I0 females; $p=0.136)$. No age-related significance was detected between the 2 groups (Group I: 63.93 years; Group 2: 59.67 years; $p=0.107$ )

In group I, radiological imaging after neoadjuvant therapy showed progression under treatment in 3 patients. The remain-

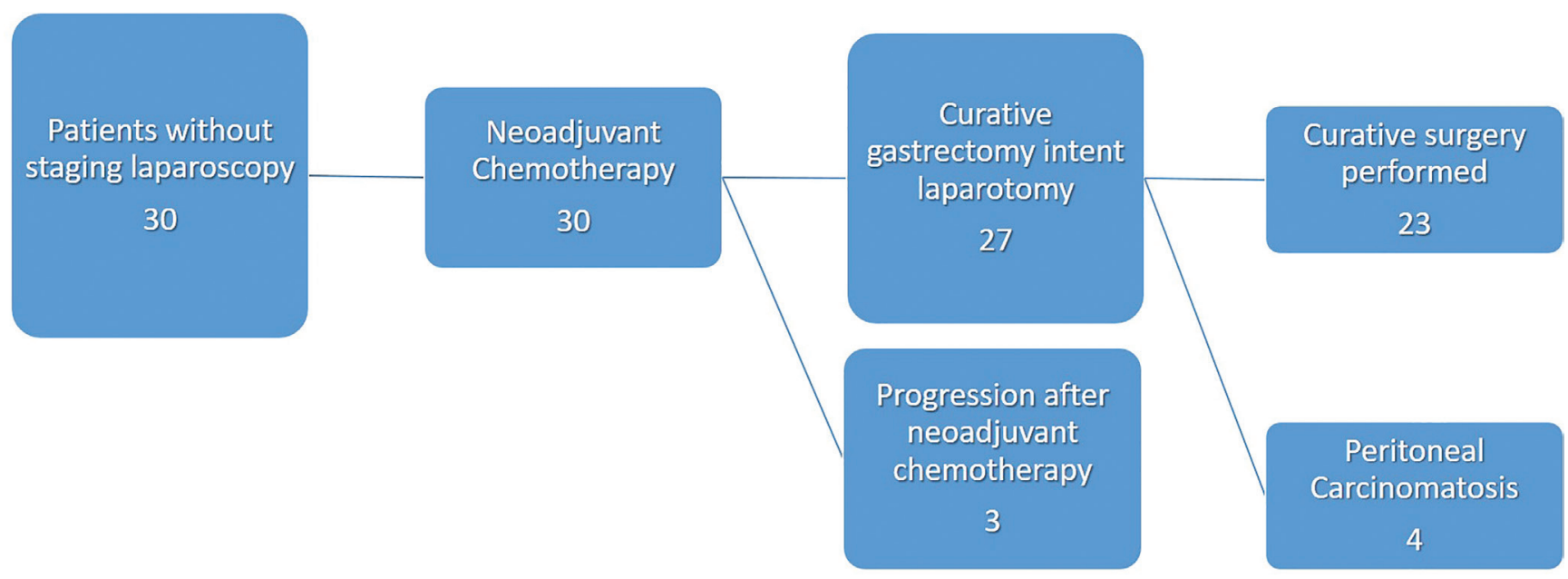




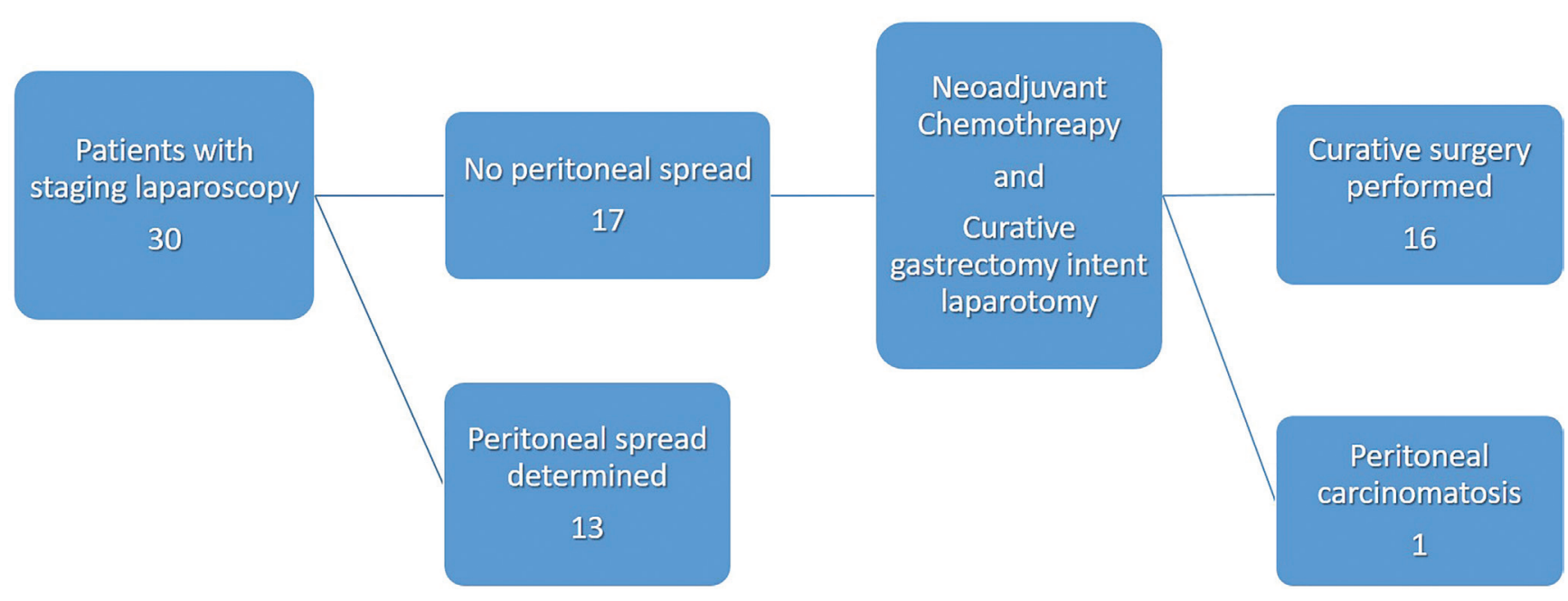

FIGURE 2. Patients with staging laparoscopy

ing 27 patients in this group were operated on and peritoneal carcinomatosis was detected in 4 patients (Figure I).

Thirteen of 30 patients in group 2 showed peritoneal spread of cancer. Ten of these 13 patients had peritoneal implants and 3 had malignant cells in the peritoneal lavage precipitate. Seventeen patients who had no peritoneal spread on laparoscopy were operated on after neoadjuvant chemotherapy. The peritoneal spread was determined during gastrectomy operation in I patient (Figure 2).

\section{DISCUSSION}

Chemotherapy and radiotherapy regimens are added to radical gastric and lymph node resection procedures to provide an adjuvant effect in gastric cancer treatment protocols $(5,6)$. In the treatment protocol, adjuvant therapies are added only if patients have poor prognostic factors such as advanced tumor invasion, lymph node metastasis, and lymphovascular invasion (7).

Neoadjuvant and adjuvant (perioperative) chemotherapy is a relatively new concept in the treatment of gastric cancer. This option is offered for $\geq \mathrm{T} 2$ tumors without distant organ or peritoneal metastasis as per the guidelines (8). It has been proposed that neoadjuvant therapy has more advantages than adjuvant therapy in our study. Adjuvant chemotherapy is delayed due to feeding problems and a prolonged recovery period, and treatment is often not completed in the postoperative period. Preoperative neoadjuvant chemotherapy is tolerated better than postoperative adjuvant chemotherapy. Although there are no difficulties and problems of tolerance in postoperative chemotherapy, there are other troubles with respect to the effectiveness of the treatment, such as surgical dissection causing deterioration of vascularity in the remaining tissues after resection. This effect causes a lower distribution of adjuvant chemotherapeutics in these tissues.

Neoadjuvant chemotherapy seems to be more successful in this context $(3,4)$. Neoadjuvant chemotherapy contributes to the achievement of the RO resection goal by decreasing the tumor and lymph node staging. Further, according to the response of the tumor to the treatment, information about the efficacy of the chemotherapeutic can be obtained, with which the transition to effective chemotherapy regimens are easily achieved if the treatment response is not enough.

Stomach cancer can metastasize to a wide variety of organs and/or structures (9). The key point for neoadjuvant chemotherapy is the distant location of a solid organ and the status of peritoneal metastasis at the beginning of treatment. Although preoperative radiological and/or nuclear medicine methods are very reliable for distant solid organ metastases, it is difficult to detect small peritoneal implants by these methods.

The sensitivity of contrast-enhanced multi-slice spiral CT and Positron Emission Tomography are reported in the literature as $25 \%-100 \%$ and $58 \%-100 \%$ respectively, for detecting peritoneal metastasis. Unfortunately, these values are decreased in the presence of small $(<5 \mathrm{~mm})$ peritoneal metastases $(10, \mathrm{II})$.

In this study, staging laparoscopy determined peritoneal metastasis in $43 \%$ of radiologically negative patients. The clinical stage and treatment protocol changed in these 13 patients by staging laparoscopy. This rate of change was reported as $48 \%$ by Shelat et al. (II)3 RI resections and I R2 resection and as 37.8\% by Muntean et al. (I2).

In group I, 3 metastatic patients were detected after neoadjuvant chemotherapy by preoperative CT, and 4 patients were detected with peritoneal metastasis after neoadjuvant chemotherapy during the operation. In these 4 patients, radiological images could not determine the peritoneal spreads due to their small dimensions (in millimeters) just before the operation. No clear comment was made on their peritoneal status prior to initiating neoadjuvant therapy for 10 patients.

In group 2, the progression despite administration of chemotherapy was determined in I patient. We concluded that this chemotherapy regimen does not have sufficient anti-tumoral activity. With this information, missed effective drugs were identified and the chemotherapy protocol was reorganized. 
When the results of this study were interpreted, 3 choices regarding the treatment suitable for 7 patients without curative gastrectomy in group 2 were put forward; A. Minimal response to treatment, B. Stable disease under treatment, C. Progression under treatment. It was decided that this question could not be answered with absolute accuracy with the present data. The effects of the applied chemotherapy regimens on the tumors were unknown. Unfortunately, the treatment protocols were revised without this information for these 7 patients.

The present study had several potential limitations. The major limitations were the retrospective design and small sample size. Another limitation was the lack of polymerase chain reaction testing, which is a more sensitive method for the evaluation of abdominal washing fluids.

Peritoneal metastasis is not uncommon in gastric cancer. When clinical staging is performed at the beginning of the treatment and if the evaluation of peritoneal status is made only by radiological examinations, the treatment may be started with an incorrect low-grade diagnosis. With the information in the previous literature and the results obtained from our study, we can conclude that the probability of error in the clinical staging is about $40 \%$. Staging laparoscopy prior to neoadjuvant chemotherapy can make a significant contribution in the multidisciplinary treatment of gastric cancer.

Ethics Committee Approval: Ethics committee approval was received for this study from Dokuz Eylul University Ethical Committee. (Approval Date: 23.01.2019, Approval Number: 2019/02-05).

Informed Consent: Informed consent was obtained from the patients who participated in this study.

Peer-review: Externally peer-reviewed.

Author contributions: Concept - S.D., K.A.; Design - S.D., C.A.; Supervision - K.A., M.Ü.; Resource - M.Ü., K.A.; Materials - C.A., M.Ü.; Data Collection and/or Processing - S.D.; Analysis and/or Interpretation - S.D., C.A., K.A., M.Ü.; Literature Search - S.D.; Writing - S.D.; Critical Reviews - K.A., M.Ü., C.A.

Acknowledgements: We would like to show our gratitude to all the official members of the Upper GI Oncology Council at Dokuz Eylul University for their contribution to this study.
Conflict of Interest: The authors have no conflicts of interest to declare.

Financial Disclosure: The authors declared that this study has received no financial support.

\section{REFERENCES}

I. Ferlay J, Soerjomataram I, Dikshit R, Eser S, Mathers C, Rebelo M, et al. Cancer incidence and mortality worldwide: Sources, methods and major patterns in GLOBOCAN 2012. Int J Cancer 2015; I36: E359-86. [CrossRef]

2. Strong VE, Song KY, Park CH, Jacks LM, Gonen M, Shah M, et al. Comparison of gastric cancer survival following $\mathrm{RO}$ resection in the United States and Korea using an internationally validated nomogram. Ann Surg 20I0; 251: 640-6. [CrossRef]

3. Cunningham D, Allum WH, Stenning SP, Thompson JN, Van de Velde $\mathrm{CJH}$, Nicolson $\mathrm{M}$, et al. Perioperative chemotherapy versus surgery alone for resectable gastroesophageal cancer. N Engl J Med 2006; 355: II-20. [CrossRef]

4. Shapiro J, van Lanschot JJB, Hulshof MCCM, van Hagen P, van Berge Henegouwen MI, Wijnhoven BPL, et al. Neoadjuvant chemoradiotherapy plus surgery versus surgery alone for oesophageal or junctional cancer (CROSS): long-term results of a randomised controlled trial. Lancet Oncol 2015; I6: 1090-8. [CrossRef]

5. Sakuramoto S, Sasako M, Yamaguchi T, Kinoshita T, Fujii M, Nashimoto A, et al. Adjuvant Chemotherapy for Gastric Cancer with S-I, an Oral Fluoropyrimidine. N Engl J Med 2007; 357: 18I0-20. [CrossRef]

6. Sasako M, Sakuramoto S, Katai H, Kinoshita T, Furukawa H, Yamaguchi T, et al. Five-Year Outcomes of a Randomized Phase III Trial Comparing Adjuvant Chemotherapy With S-I Versus Surgery Alone in Stage II or III Gastric Cancer. J Clin Oncol 2010; 29: 4387-93. [CrossRef]

7. Kaya V, Fidan Korcum A, Yıldırım M, Gamze Aksu M, Mutlu H, Șirin Özdemir B, et al. Prognostic factors in gastric cancer patients treated with adjuvant chemoradiotherapy. ACU Sağlık Bil Derg 2015: 214.

8. Lisa Gurski N, McMillian N, Lenora Pluchino MA, Farjah F, Gerdes H, Gibson M, et al. NCCN Guidelines Version 2.2018 Panel Members Gastric Cancer [Internet]. [cited 2018 Dec 22]. Available from: URL: https://www.nccn.org/professionals/physician_gls/pdf/gastric. pdf.

9. Ozdemir L, Ozdemir B, Polat Y. Endobronchial Metastasis of Gastric Adenocarcinoma. Cyprus J Med Sci 20l6; I: 36-6. [CrossRef]

10. Patel CM, Sahdev A, Reznek RH. CT, MRI and PET imaging in peritoneal malignancy. Cancer Imaging 20II; II: 123-39. [CrossRef]

II. Shelat VG, Thong JF, Seah M, Lim KH. Role of staging laparoscopy in gastric malignancies - our institutional experience. World J Gastrointest Surg 2012; 4: 214-9. [CrossRef]

12. Muntean $\vee$, Mihailov A, lancu C, Toganel R, Fabian O, Domsa I, et al. Staging laparoscopy in gastric cancer. Accuracy and impact on therapy. J Gastrointestin Liver Dis 2009; 18: 189-95. 